\title{
Unilateral Versus Bilateral Prostatic Arterial Embolization for Lower Urinary Tract Symptoms in Patients with Prostate Enlargement
}

\author{
Tiago Bilhim • João Pisco $\cdot$ Hugo Rio Tinto $\cdot$ Lúcia Fernandes $\cdot$ \\ Luís Campos Pinheiro - Marisa Duarte · José A. Pereira • \\ António G. Oliveira · João O’Neill
}

Received: 31 July 2012/Accepted: 15 November 2012/Published online: 12 December 2012

(C) Springer Science+Business Media New York and the Cardiovascular and Interventional Radiological Society of Europe (CIRSE) 2012

\begin{abstract}
Purpose This study was designed to compare baseline data and clinical outcome between patients with prostate enlargement/benign prostatic hyperplasia $(\mathrm{PE} / \mathrm{BPH})$ who underwent unilateral and bilateral prostatic arterial embolization (PAE) for the relief of lower urinary tract symptoms (LUTS).

Methods This single-center, ambispective cohort study compared 122 consecutive patients (mean age 66.7 years) with unilateral versus bilateral PAE from March 2009 to December 2011. Selective PAE was performed with 100and $200-\mu \mathrm{m}$ nonspherical polyvinyl alcohol (PVA) particles by a unilateral femoral approach.
\end{abstract}

This work reflects in part the work from Bilhim T. Embolização das artérias prostáticas no tratamento da hiperplasia benigna da próstata. $\mathrm{PhD}$ thesis, Faculdade de Ciências Médicas, Universidade Nova de Lisboa, Lisbon, Portugal, May 2011.

T. Bilhim $(\bowtie) \cdot$ J. O’Neill

Departamento Universitário de Anatomia, Faculdade de Ciências Médicas (FCM), Universidade Nova de Lisboa (UNL), Campo dos Mártires da Pátria, 130, Lisbon 1169-056, Portugal

e-mail: tiagobilhim@hotmail.com

T. Bilhim - J. Pisco $\cdot$ H. Rio Tinto $\cdot$ L. Fernandes - M. Duarte J. A. Pereira

Interventional Radiology, Saint Louis Hospital, Rua Luz

Soriano, 182, Lisbon 1200-249, Portugal

T. Bilhim · H. Rio Tinto $\cdot$ L. Fernandes

Departamento Universitário de Radiologia, Faculdade de Ciências Médicas (FCM), Universidade Nova de Lisboa (UNL), Campo dos Mártires da Pátria, 130, Lisbon 1169-056, Portugal

L. Campos Pinheiro

Departamento Universitário de Urologia, Faculdade de Ciências Médicas (FCM), Universidade Nova de Lisboa (UNL), Campo dos Mártires da Pátria, 130, Lisbon 1169-056, Portugal
Results Bilateral PAE was performed in $103(84.4 \%)$ patients (group A). The remaining 19 (15.6\%) patients underwent unilateral PAE (group B). Mean follow-up time was 6.7 months in group A and 7.3 months in group B. Mean prostate volume, PSA, International prostate symptom score/ quality of life (IPSS/QoL) and post-void residual volume (PVR) reduction, and peak flow rate (Qmax) improvement were $19.4 \mathrm{~mL}, 1.68 \mathrm{ng} / \mathrm{mL}, 11.8 / 2.0$ points, $32.9 \mathrm{~mL}$, and $3.9 \mathrm{~mL} / \mathrm{s}$ in group A and $11.5 \mathrm{~mL}, 1.98 \mathrm{ng} / \mathrm{mL}, 8.9 / 1.4$ points, $53.8 \mathrm{~mL}$, and $4.58 \mathrm{~mL} / \mathrm{s}$ in group B. Poor clinical outcome was observed in $24.3 \%$ of patients from group A and $47.4 \%$ from group B $(p=0.04)$.

Conclusions PAE is a safe and effective technique that can induce $48 \%$ improvement in the IPSS score and a prostate volume reduction of $19 \%$, with good clinical outcome in up to $75 \%$ of treated patients. Bilateral PAE seems to lead to better clinical results; however, up to $50 \%$

L. Campos Pinheiro

Urology Department, São José Hospital, Centro Hospitalar de Lisboa Central, Rua José António Serrano, Lisbon 1150-199, Portugal

\section{A. G. Oliveira}

Biostatistics Department, Faculdade de Ciências Médicas (FCM), Universidade Nova de Lisboa (UNL), Campo dos Mártires da Pátria, 130, Lisbon 1169-056, Portugal

J. O'Neill

CEFITEC Centro de Física e Investigação Tecnológica - FCTUNL, Quinta da Torre, Caparica 2825-516, Portugal 
of patients after unilateral PAE may have a good clinical outcome.

Keywords Prostatic arterial embolization - Unilateral . Benign prostatic hyperplasia - Lower urinary tract symptoms

Preliminary studies have shown that prostatic arterial embolization (PAE) for the relief of lower urinary tract symptoms (LUTS) in patients with prostate enlargement/ benign prostatic hyperplasia $(\mathrm{PE} / \mathrm{BPH})$ is a promising, new, minimally invasive, image-guided therapy [1-4]. However, PAE is a technically challenging procedure with unilateral embolization and technical failure reported rates of approximately $10-15$ and $7 \%$, respectively [1-4]. To perform the procedure in a safe manner without nontarget embolization to periprostatic tissues, precise knowledge of the male pelvic and PA anatomy and imaging findings is required [5-7].

One of the reported series [2, 4] included one patient with unilateral embolization (with almost $20 \%$ reduction in prostate volume and $11 \%$ PSA reduction) and another with bilateral PAE (with almost $50 \%$ reduction in prostate volume and $82 \%$ PSA reduction). Both patients had significant clinical relief. Another series [3] showed that unilateral PAE might lead to moderate clinical relief with $8 \%$ prostate volume reduction and $18 \%$ reduction in PSA. However, the preliminary case report described a patient who underwent unilateral PAE with $38 \%$ prostate volume reduction, $90 \%$ decrease in PSA, and significant clinical relief [1].

We found no study to date that has compared the results between unilateral versus bilateral PAE in the treatment of LUTS in patients with PE/BPH. The purpose of this study was to compare baseline data and clinical outcome between patients who underwent unilateral and bilateral PAE for the relief of LUTS.

\section{Materials and methods}

This single-center, ambispective, cohort study compared 122 consecutive patients who underwent unilateral versus bilateral PAE from March 2009 to December 2011 (mean patient age $66.7 \pm 7.2$ years) with $\mathrm{PE} / \mathrm{BPH}$ who underwent PAE for the relief of LUTS or for the treatment of acute urinary retention (AUR). The institutional review board approved the study, and a signed informed consent was obtained from all patients.

All patients were evaluated by clinical observation measuring the severity of LUTS with the international prostate symptom score (IPSS) and the quality of life
(QoL) related to LUTS. Erectile function was evaluated with the International Index of Erectile Function (IIEF). Prostate volume (PV) assessment was performed by digital rectal examination (DRE) and 2D-transrectal ultrasound (TRUS) using the ellipsoid formula $\pi / 6 \times$ (transverse diameter $\times$ anteroposterior diameter $\times$ sagittal diameter). PSA was measured in all patients. Uroflowmetry measuring the maximum flow rate (Qmax) and the postvoid residual volume (PVR) was performed in all patients who were not experiencing AUR. Pre-procedural sectional imaging planning with CT angiography (CTA) to evaluate the feasibility of PAE and study the pelvic arterial anatomy was performed in all patients [5-7]. If the PSA level was greater than $4 \mathrm{ng} / \mathrm{mL}$ or when the DRE or TRUS findings were suspicious, prostatic biopsy was performed to exclude malignancy.

Inclusion criteria for PAE included male patients, age $>40$ years, prostate volume $>30 \mathrm{cc}$, and diagnosis of PE/ $\mathrm{BPH}$ with moderate to severe LUTS refractory to medical treatment for at least 6 months (IPSS >18 and/or QoL >3) or under acute urinary retention refractory to medical therapy. All patients were receiving medical therapy with one alpha-1-adrenergic receptor antagonist (alfuzosin $10 \mathrm{mg}$, Ratiopharm, once daily; doxazosin $4 \mathrm{mg}$, Cardura Gits, Pfizer, once daily; or tamsulosin $0.4 \mathrm{mg}$, Ratiopharm, once daily). Thirty-one patients $(25.4 \%)$ also were medicated with 5-alpha-reductase inhibitors (finasteride $5 \mathrm{mg}$, Proscar, Merck Sharp \& Dohme, once daily; or dutasteride $0.5 \mathrm{mg}$, Avodart, GlaxoSmithKline, once daily). Four patients had previous prostatic surgery (TURP) years before. Sixteen patients $(13.1 \%)$ were experiencing AUR before PAE. Exclusion criteria included malignancy, large bladder diverticula $(>5 \mathrm{~cm})$, large bladder stones $(>2 \mathrm{~cm})$, chronic renal failure (glomerular filtration rate $<60 \mathrm{~mL} / \mathrm{min}$; serum creatinine $>1.2 \mathrm{mg} / \mathrm{dl}$ ), tortuosity and advanced atherosclerosis of iliac and/or prostatic arteries (PAs) on pre-procedural CTA (on the basis of visual evaluation by the interventional radiologists), active urinary tract infection, and unregulated coagulation parameters.

All patients were evaluated with IPSS/QoL, IIEF, PV, PSA, Qmax, and PVR measurements. Baseline data were assessed, and response to treatment was evaluated 1, 3, 6, and 12 months after PAE.

Our procedure protocol has been described previously [3]. We asked patients to stop all prostatic medication 1 week before embolization, if they were able to tolerate it. If the procedure was clinically successful, patients were told to abandon all prostatic medication after PAE. Two days before PAE, in the day of PAE and on the following 10 days, patients were medicated with an acid-suppressing drug (omeprazole $20 \mathrm{mg}$, Bluepharma, once daily), an antiinflammatory (naproxen 1,000 mg, Naprosyn, Roche, twice daily), and an antibiotic (ciprofloxacin $750 \mathrm{mg}$, Jaba, 
twice daily). Patients were admitted to the hospital on the morning of the procedure and were discharged the same day if no complications occurred. During PAE analgesic and anti-inflammatory drugs were given intravenously (ketorolac $30 \mathrm{mg}$ and metamizol $2 \mathrm{~g}$ ).

A unilateral femoral approach (under local anesthesia) was performed with a $5 \mathrm{~F} 11-\mathrm{cm}$-long hydrophilic sheath (Cordis, NJ, USA). A pigtail catheter (F5, Cordis) or the Roberts uterine artery catheter (Cook, Bloomington, IN) was introduced in the femoral artery with a $0.035^{\prime}$ hydrophilic guidewire (Terumo, Tokyo, Japan) to perform the crossover. Afterwards, the Waltman loop was reformed on the Roberts uterine artery catheter that was used to catheterize both hypogastric arteries. The catheter tip was left in the proximal part of the anterior division of the hypogastric arteries and digital subtraction angiography (DSA) using nonionic optiray contrast medium (Ioversol, $350 \mathrm{mg} \mathrm{I} / \mathrm{ml}$; Covidien, Dublin, Ireland; $6 \mathrm{~mL}, 3 \mathrm{~mL} / \mathrm{s}, 3$ frames/s) was performed with ipsilateral anterior oblique projection $\left(35^{\circ}\right)$ and caudal-cranial angulation $\left(10^{\circ}\right)$. Afterwards, the prostatic vessels were selectively catheterized with a 2.4-2.7 F-microcatheter (Cantata, Cook; Progreat, Terumo, Tokyo, Japan) and a $0.016^{\prime}$ guidewire (Sagitta, Cook; Glidewire GT, Terumo). Selective prostatic artery DSA was performed manually with 3-5 mL of contrast volume in neutral and ipsilateral anterior oblique $\left(35^{\circ}\right)$ and caudal-cranial angulation $\left(10^{\circ}\right)$ projection. Selective PAE was performed with 100 - and $200-\mu \mathrm{m}$ nonspherical polyvinyl alcohol (PVA) particles (Cook). The endpoint for embolization was interruption of the arterial flow to the prostate, reflux, and prostate gland opacification.

Procedure time (starting with femoral puncture access and finished after removal of the catheter) and fluoroscopy time were measured. Pain was measured using a visual analogue scale (VAS; $0=$ sensation of no pain; $10=$ worst pain), by verbal questioning and written questionnaires during and in the 6-8 $\mathrm{h}$ following PAE.

Postembolization symptoms and complications were registered and classified according to the quality improvement guidelines for percutaneous transcatheter embolization [8]. All patients were evaluated by clinical and physical examination with pain assessment in the day after PAE and weekly in the following months.

Poor clinical outcome after PAE was considered when one criterion was met: IPSS $\geq 20$ and/or reduction $<25 \%$; QoL $\geq 4$ and/or reduction $<1$; Qmax improvement $<2.5 \mathrm{~mL} / \mathrm{s}$; additional treatments required (need of prostatic medication or surgery due to persisting severe LUTS) $[9,10]$.

For comparisons of baseline and outcome variables between the two groups, the paired $t$ test and Fisher's exact tests were used. For comparisons of the change from baseline of outcome variables between groups, an analysis of covariance (ANCOVA) with adjustment for age was used. For the comparison of poor clinical outcome, logistic regression adjusted for age was used. Reported values are means and standard deviations. Statistically significant differences were assumed at $p<0.05$. Stata software release 12 (Stata Corporation, College Station, TX) was used for all statistical analyses.

\section{Results}

Bilateral PAE was performed in $103(84.4 \%)$ patients (group A). The remaining 19 (15.6\%) patients underwent unilateral PAE (group B). Mean follow-up time was 6.7 months in group A ( 1 year in 30 patients; 6 months in 43 patients; 3 months in 23 patients; and 1 month in 7 patients) and 7.3 months in group B (1 year in 10 patients; 6 months in 2 patients; 3 months in 7 patients). Most patients who underwent unilateral PAE had significant atherosclerotic lesions and tortuosity of the iliac and prostatic arteries or prostatic arteries that arose from the superior vesical artery with angled origins and tortuous trajectory (Figs. 1, 2, 3).

Patients in group B were significantly older than patients in group A (Table 1); however, no other statistically significant differences were found in the baseline data between groups. Overall, mean procedural time was 83.7 min (range 26-182) with a mean fluoroscopy time of 27.8 min (range 8-61). In group A, the mean procedural time was $67.3 \pm 30.9 \mathrm{~min}$, and it was $96.3 \pm 26.4 \mathrm{~min}$ in group B $(p=0.0007)$. The mean fluoroscopy time was $18.1 \pm 12.9 \mathrm{~min}$ in group $\mathrm{A}$ and $34.0 \pm 10.3 \mathrm{~min}$ in group B $(p<0.0001)$.

Mean procedural pain score was 3.1 and 0.2 points after PAE (before discharge), with 15 (12.2\%) patients discharged the following day (inpatients) because of personal preference (the remaining patients were treated as outpatients and were discharged 6-8 $\mathrm{h}$ after PAE).

As minor complications, 12 (9.8\%) patients had urinary tract infections after embolization treated with antibiotics (10 patients from group $\mathrm{A}-9.7 \%$ and 2 patients from group B-10.5\%), transient hematuria in 16 (13.1\%) patients, transient hemospermia in $8(6.6 \%)$ patients, and transient rectal bleeding in $10(8.2 \%)$ patients that disappeared spontaneously without any treatment during the first 2 weeks. There were two cases of balanoprostatites (1.6\%) and nine inguinal hematomas $(7.4 \%)$ that required no additional treatment. Two patients had acute urinary retention after PAE; for relief, a temporary bladder catheter was placed at the time for a couple of hours and the patients were able to void spontaneously before discharge. No other minor or major complications were observed. Sixteen patients were under AUR before PAE (13 from 


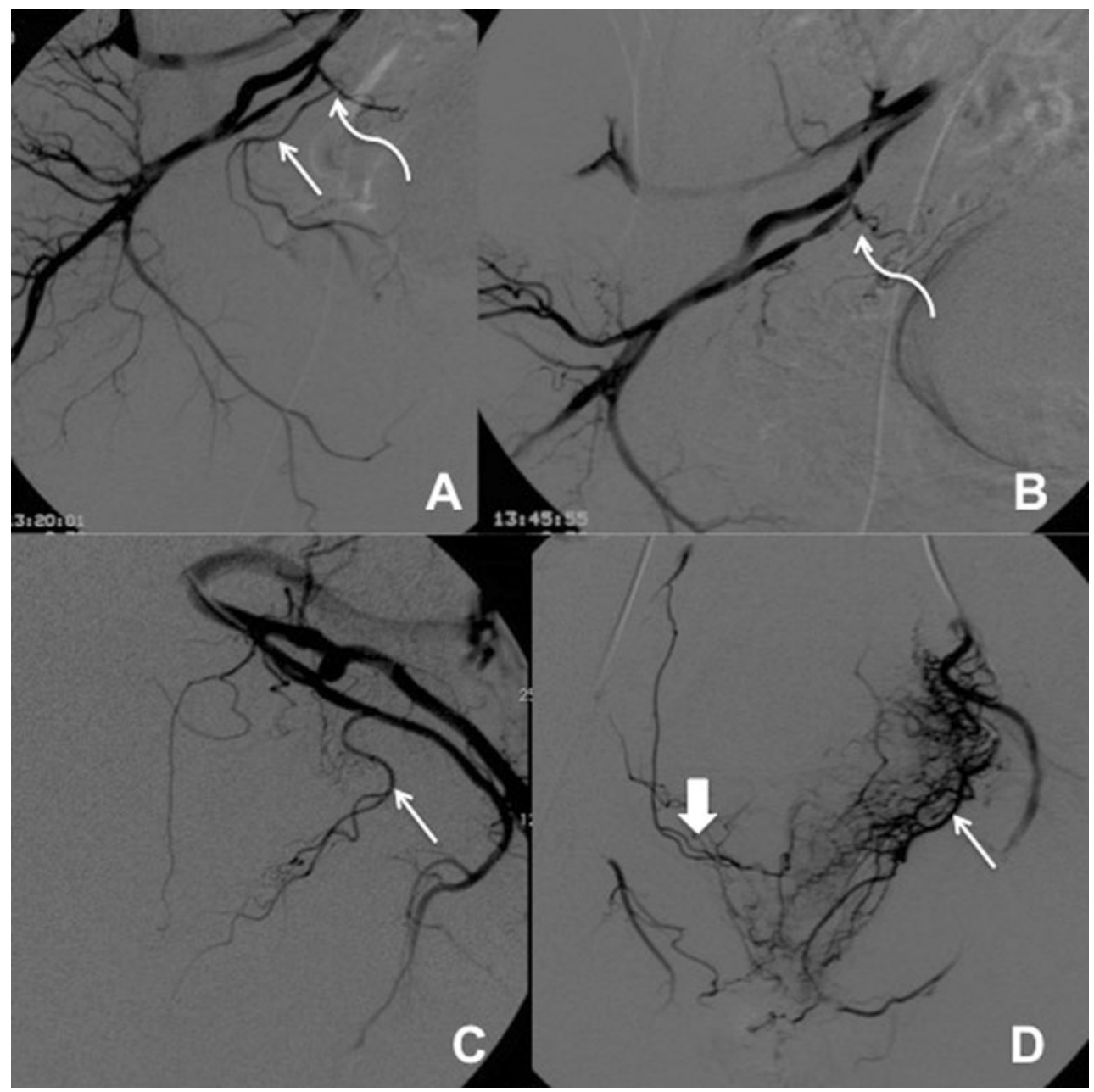

Fig. 1 Unilateral prostatic arterial embolization (PAE). A Right pelvic side, digital subtraction angiography (DSA) with ipsilateral anterior oblique projection $\left(35^{\circ}\right)$ and caudal-cranial angulation $\left(10^{\circ}\right)$. The prostatic artery (straight arrow) arises from the superior vesical artery (curved arrow) with an angled origin $\left(90^{\circ}\right)$. B Right pelvic side, DSA with ipsilateral anterior oblique projection $\left(35^{\circ}\right)$ and caudalcranial angulation $\left(10^{\circ}\right)$. After various attempts to selective

group A and 3 from group B) and all were able to remove the bladder catheter and urinate spontaneously 15-60 days after PAE.

Overall, there was a mean prostate volume reduction of $18.2 \pm 22.1 \mathrm{~mL}(19.2 \%, p<0.0001)$, a mean PSA reduction of $1.73 \pm 4.33 \mathrm{ng} / \mathrm{mL}(30.9 \%, p<0.0001)$, a mean IPSS/QoL improvement of $11.3 \pm 8.74 / 1.93 \pm 1.52$ points $(44.8 \% / 44.2 \%, p<0.0001)$, a mean $\mathrm{Qmax}$ improvement of $4.00 \pm 4.75 \mathrm{~mL} / \mathrm{s}(65 \%, p<0.0001)$, and a mean PVR reduction of $35.9 \pm 93.2 \mathrm{~mL}(0.5 \%$, $p=0.002)$. The IIEF improved $1.55 \pm 6.44$ points $(23.6 \%, p=0.017)$.

There was a statistically significant difference between groups regarding clinical outcome with $47.4 \%$ of patients from group B and $24.3 \%$ of patients from group A having catheterize the prostatic artery with a microcatheter, the artery was dissected (curved arrow) and is no longer visible in the control angiogram. C Left pelvic side, DSA with ipsilateral anterior oblique projection $\left(35^{\circ}\right)$ and caudal-cranial angulation $\left(10^{\circ}\right)$ showing the left prostatic artery (straight arrow). D Left prostatic artery DSA (straight arrow) in neutral position showing anastomoses to the contralateral prostatic artery (solid arrow) poor clinical outcome (23.1\% difference, $p=0.04)$. Age was a confounder and after adjusting for age, this trend was maintained but did not reach statistical significance. There were no statistically significant differences in the improvement rates of most evaluated parameters after PAE between groups (Table 2). The PV volume reduced more $7.9 \mathrm{~mL}$, the IPSS 2.9 points, and the QoL 0.6 points in group A. The PSA, Qmax, and PVR improvements after PAE did not differ significantly between groups.

There were $34(27.9 \%)$ patients with poor clinical outcome after PAE ( 25 from group A-24.3\% and 9 from group B-47.4\%). Six patients underwent subsequent prostatic surgery (5 from group A-4.9 \% and 1 from group B-5.3\%), whereas 20 patients resumed prostatic medication and remained under observation (13 from 
Fig. 2 Atherosclerotic lesions that may lead to unilateral PAE. A CTA, Sagittal MIP reformat of the left pelvic side showing an occlusion of the internal pudendal artery (curved arrow) above the prostatic artery origin (straight arrow). B Left pelvic side, DSA with ipsilateral anterior oblique projection $\left(35^{\circ}\right)$ and caudal-cranial angulation $\left(10^{\circ}\right)$ showing the catheter tip (solid arrow) near the occlusion (curved arrow). There is left prostatic artery (straight arrow) retrograde opacification through the vesical arteries (dotted arrows). C Left pelvic side, DSA with ipsilateral anterior oblique projection $\left(35^{\circ}\right)$ and caudal-cranial angulation $\left(10^{\circ}\right)$ showing multiple atherosclerotic stenoses of the internal pudendal artery (curved arrows) and a small and tortuous prostatic artery (straight arrow). D CTA, 3D volume rendering reformat of the right pelvic side showing multiple atherosclerotic stenoses of the internal pudendal artery (curved arrows) that may lead to technical difficulties. E Right pelvic side, DSA with ipsilateral anterior oblique projection $\left(35^{\circ}\right)$ and caudal-cranial angulation $\left(10^{\circ}\right)$. Multiple atherosclerotic stenoses (curved arrows) of the internal iliac anterior division and of the origin of the prostatic artery (straight arrow) and obturator artery (solid arrow)

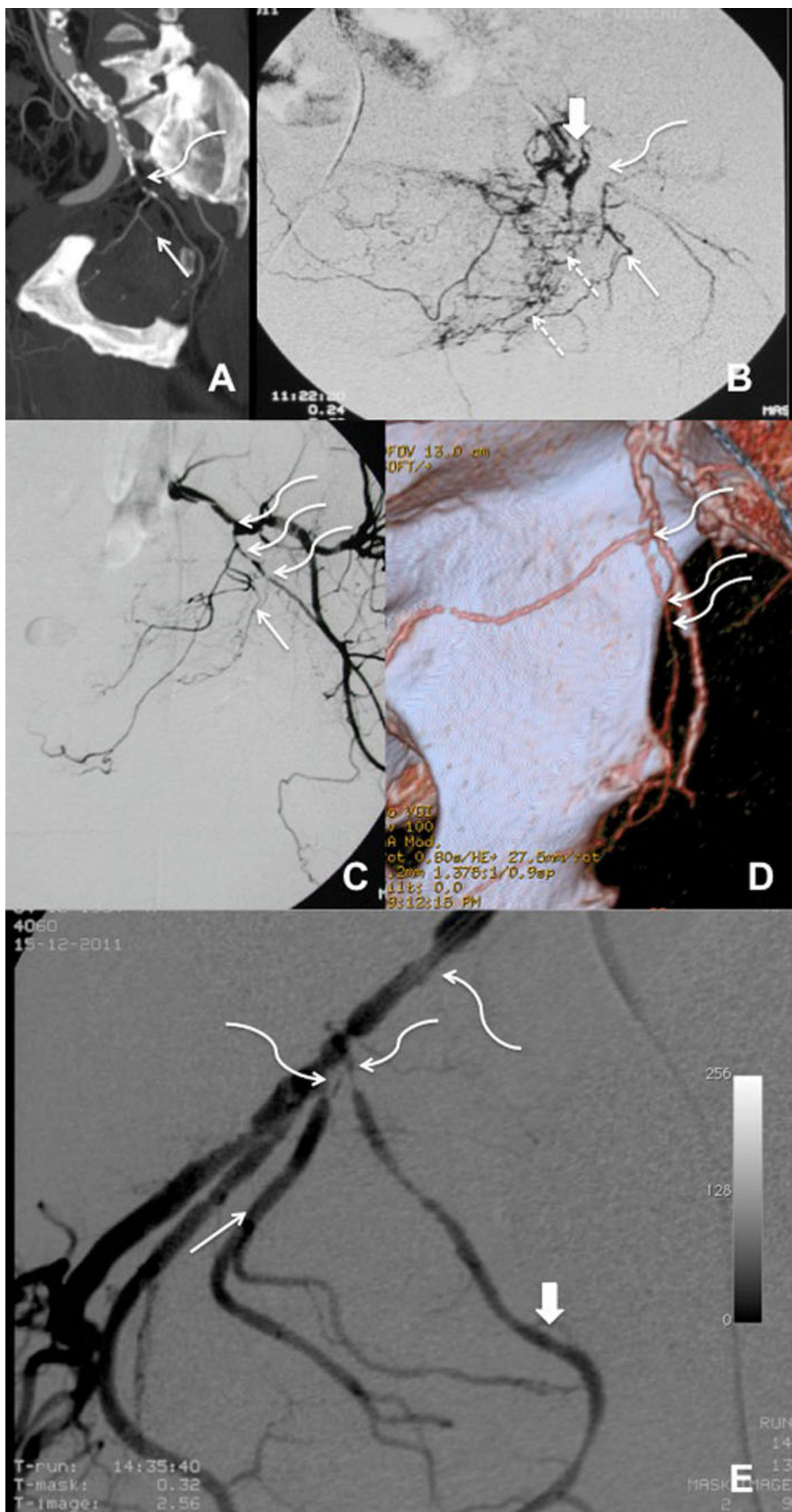



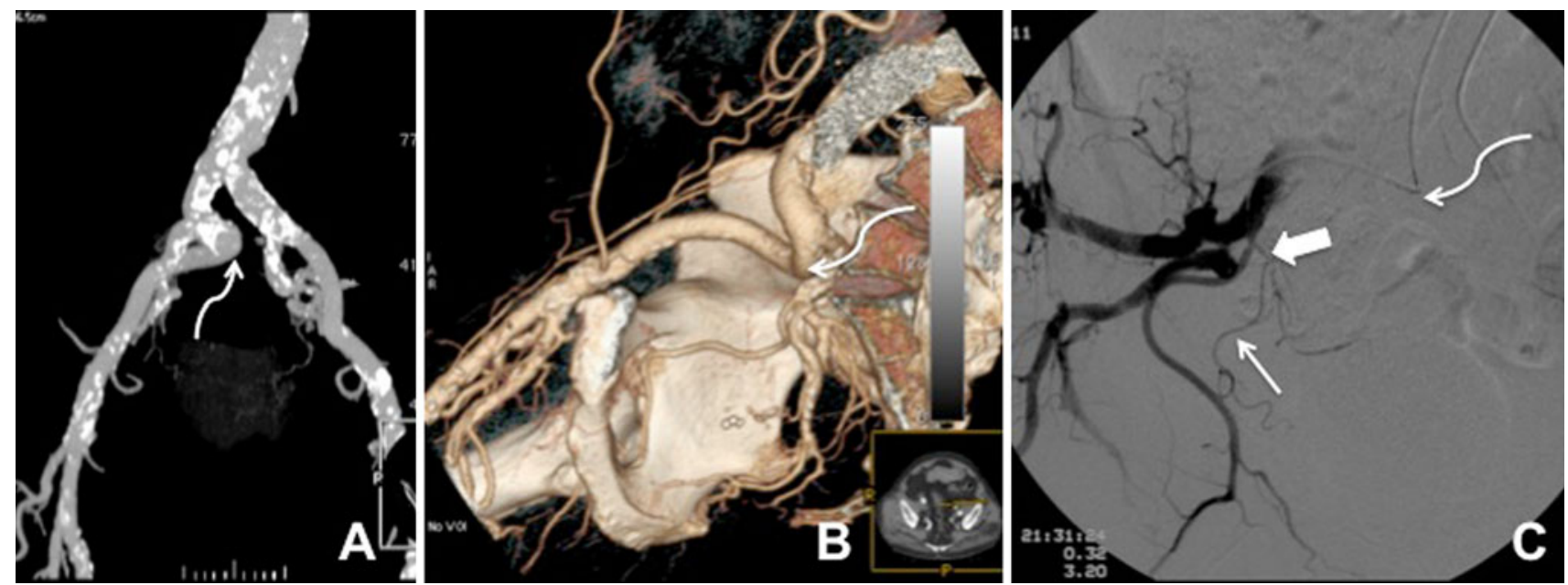

Fig. 3 Tortuosity and atherosclerosis of the right pelvic side arteries (curved arrows) leading to unilateral PAE. A CTA, 3D MIP reformat. B CTA, 3D volume rendering reformat. C Right pelvic side, DSA with ipsilateral anterior oblique projection $\left(35^{\circ}\right)$ and caudal-cranial angulation $\left(10^{\circ}\right)$. Note the catheter kinking due to tortuosity of the right pelvic arteries (curved arrow). The prostatic artery (straight arrow) has a tortuous trajectory, small diameter and arises from the superior vesical artery with an angled origin (solid arrow)

Table 1 Baseline data between groups (group A, bilateral PAE; group B, unilateral PAE)

\begin{tabular}{llll}
\hline Baseline data & Group A $(n=103)$ & Group B $(n=19)$ & $P$ value \\
\hline Mean age (mean $\pm \mathrm{SD}$, year) & $65.8 \pm 6.9$ & $71.3 \pm 1.7$ & 0.002 \\
PV (mean $\pm \mathrm{SD}, \mathrm{mL})$ & $84.1 \pm 38.5$ & $75.8 \pm 30.8$ & 0.38 \\
PSA (mean $\pm \mathrm{SD}, \mathrm{ng} / \mathrm{mL})$ & $5.6 \pm 5.5$ & $7.5 \pm 9.3$ & 0.22 \\
IPSS (mean $\pm \mathrm{SD}$, points) & $23.1 \pm 6.8$ & $21.9 \pm 7.0$ & $3.9 \pm 0.9$ \\
QoL (mean $\pm \mathrm{SD}$, points) & $4.2 \pm 0.9$ & $8.4 \pm 3.1$ & 0.54 \\
Qmax (mean $\pm \mathrm{SD}, \mathrm{mL} / \mathrm{s})$ & $8.5 \pm 4.2$ & $116.2 \pm 86.9$ & $31.6(n=6)$ \\
PVR (mean $\pm \mathrm{SD}, \mathrm{mL})$ & $93.9 \pm 92.3$ & $26.3(n=5)$ & 0.90 \\
5-ARI medication $(\%)$ & $24.3(n=25)$ & $15.8(n=3)$ & 0.37 \\
Diabetic $(\%)$ & $15.5(n=16)$ & $12.6(n=13)$ & 0.32 \\
AUR $(\%)$ & & 0.71
\end{tabular}

$S D$ standard deviation; $P V$ prostate volume; IPSS International Prostate Symptom Score; $Q o L$ quality of life; $Q \max$ maximum flow rate; $P V R$ postvoid residual volume; 5-ARI 5-alpha-reductase inhibitors; AUR acute urinary retention

Table 2 Clinical response after PAE between groups (group A, bilateral PAE; group B, unilateral PAE)

\begin{tabular}{lllll}
\hline$\Delta$ after PAE & Group A $(n=103)$ & Group B $(n=19)$ & $P$ value (unadjusted) & $P$ value (age-adjusted) \\
\hline PV (mean \pm SD, mL) & $-19.4 \pm 22.7(-20.5 \%)$ & $-11.5 \pm 17.7(-11.9 \%)$ & 0.21 & 0.38 \\
PSA (mean $\pm \mathrm{SD}, \mathrm{ng} / \mathrm{mL})$ & $-1.7 \pm 4.4(-30.4 \%)$ & $-2.0 \pm 4.1(-26.7 \%)$ & 0.33 & 0.23 \\
IPSS (mean $\pm \mathrm{SD}$, points) & $-11.8 \pm 8.8(-46.2 \%)$ & $-8.9 \pm 8.1(-37.1 \%)$ & 0.29 & 0.55 \\
QoL (mean $\pm \mathrm{SD}$, points) & $-2.0 \pm 1.6(-46.1 \%)$ & $-1.4 \pm 1.2(-33.3 \%)$ & 0.34 & 0.66 \\
Qmax (mean $\pm \mathrm{SD}, \mathrm{mL} / \mathrm{s})$ & $+3.9 \pm 4.4(+63.9 \%)$ & $+4.6 \pm 6.5(+71.6 \%)$ & 0.66 & 0.59 \\
PVR (mean $\pm \mathrm{SD}, \mathrm{mL})$ & $-32.9 \pm 93.8(-5.6 \%)$ & $-53.8 \pm 90.6(-25.9 \%)$ & 0.90 & 0.79 \\
IIEF (mean $\pm \mathrm{SD}$, points) & $+1.6 \pm 6.6(+24.5 \%)$ & $+1.1 \pm 5.5(+18.6 \%)$ & 0.41 & 0.93 \\
Poor clinical outcome $(\%)$ & $24.3(n=25)$ & $47.4(n=9)$ & 0.04 & 0.08 \\
\hline$\Delta$ varia
\end{tabular}

$\Delta$ variation; $S D$ standard deviation; $P V$ prostate volume; IPSS International Prostate Symptom Score; $Q o L$ quality of life; $Q m a x$ maximum flow rate; $P V R$ postvoid residual volume 
Table 3 Distribution of patients with poor clinical outcome stratified according to each criterion (group A, bilateral PAE; group B, unilateral PAE)

\begin{tabular}{lll}
\hline Criteria of poor clinical outcome & $\begin{array}{l}\text { Group A } \\
(n=25 / 103 ; \\
24.3 \%)\end{array}$ & $\begin{array}{l}\text { Group B } \\
(n=9 / 19 ; \\
47.4 \%)\end{array}$ \\
\hline $\begin{array}{l}\text { IPSS } \geq 20 \text { and/or reduction } \\
\quad<25 \% \text { after PAE }\end{array}$ & $13(52 \%)$ & $6(66.7 \%)$ \\
$\begin{array}{l}\text { QoL } \geq 4 \text { and/or reduction } \\
<1 \text { after PAE }\end{array}$ & $12(48 \%)$ & $6(66.7 \%)$ \\
$\begin{array}{l}\text { Qmax improvement } \\
<2.5 \text { mL/s after PAE }\end{array}$ & $16(64 \%)$ & $5(55.6 \%)$ \\
$\begin{array}{l}\text { Need for prostatic medication } \\
\text { after PAE }\end{array}$ & $13(52 \%)$ & $7(77.8 \%)$ \\
$\begin{array}{l}\text { Need for prostatic surgery } \\
\text { after PAE }\end{array}$ & $5(20 \%)$ & $1(11.1 \%)$ \\
$\begin{array}{l}\text { No need for prostatic medication } \\
\text { or surgery after PAE }\end{array}$ & $7(28 \%)$ & $1(11.1 \%)$ \\
\hline
\end{tabular}

IPSS International Prostate Symptom Score; PAE prostatic arterial embolization; $Q o L$ Quality of life; $Q \max$ maximum flow rate

Data given in number of patients (percentages) of poor clinical outcome stratified according to each criteria in each group $(p>0.05$, Fisher's exact test)

group $\mathrm{A}-12.6 \%$ and 7 from group $\mathrm{B}-36.8 \%)$. The remaining eight patients ( 7 from group $\mathrm{A}-6.8 \%$ and 1 from group $\mathrm{B}-5.3 \%$ ) had significant IPSS and QoL improvements (requiring no additional medical or surgical therapy) but were considered to have a poor outcome based on the Qmax results (without improvement). Poor clinical outcome after PAE based on symptomatic parameters and on the need for further treatment was observed in 18 patients from group A $(17.5 \%)$ and 8 patients from group B $(42.1 \%$ ) (unadjusted $p$ value of 0.03 ; age-adjusted $p$ value of 0.2 ). Overall, based on clinical symptomatic parameters and on the need of further treatment, poor outcome after PAE was observed in $26(21.3 \%)$ patients. These differences were not statistically significant between the two groups (Table 3).

\section{Discussion}

Pelvic arterial embolization to control severe bladder or prostatic hemorrhage due to neoplasms after biopsy or surgery has been reported in the past three decades with hundreds of patients treated with success. It has proved to be a safe and effective technique to control refractory, lifethreatening bladder, or prostate bleeding. The procedure should be considered the treatment of choice, because it obviates the need for emergency surgery in severely ill patients [11-16]. Animal studies have reported that PAE is a safe technique, inducing PV reduction, prostatic infarction, and ablation without sexual dysfunction [17-19].
The first report of PAE inducing LUTS relief was in a patient with BPH (unfit for surgery) with severe prostatic bleeding requiring multiple blood transfusions, refractory to interstitial laser ablation, and external-beam radiation. PAE stopped the hemorrhage and induced significative LUTS relief, PV, and PSA reduction [1]. Based on these reports, it has been suggested that PAE might follow uterine artery embolization for fibroids [20].

The preliminary results in 16 patients with $\mathrm{PE} / \mathrm{BPH}$ and severe LUTS/experiencing acute urinary retention have shown that PAE is safe and feasible procedure, with shortand mid-term follow-up suggesting good symptom control without sexual dysfunction, associated with a reduction in PV and PSA. PAE is considered technically challenging and unilateral PAE has been reported in all of these preliminary studies [2-4]. In the present study, $15.6 \%$ of patients underwent unilateral embolization due to significant atherosclerotic lesions and tortuosity of the iliac and prostatic arteries.

All of the preliminary data results from single-center, small-sized studies. To our knowledge, we found no studies comparing unilateral and bilateral PAE in a large series of patients. We aimed to analyze retrospectively the baseline data between groups to see if there was any clinical predictor of unilateral PAE. We excluded patients with large bladder diverticula, because these are patients at high risk of poor outcome after embolization due to bladder dysfunction. Also, large bladder stones and large diverticula should be removed surgically to avoid possible complications (hematuria, bladder cancer). Age was the only baseline parameter that was statistically significantly different between groups. Older patients were at greater risk of unilateral PAE and were more likely to pose technical difficulties (probably due to greater tortuosity and atherosclerotic changes of the pelvic arteries). Although there was a greater rate of diabetic patients with unilateral embolization, this trend did not reach statistical significance. The mean procedural and fluoroscopy times were significantly larger in patients with unilateral PAE reflecting the technical difficulties (mean procedural times of approximately $1 \mathrm{~h}$ for bilateral PAE and $1.5 \mathrm{~h}$ for unilateral PAE; mean fluoroscopy times under $20 \mathrm{~min}$ for bilateral PAE and approximately $30 \mathrm{~min}$ for unilateral PAE).

We prospectively compared clinical outcome between patients with unilateral and bilateral embolization. As expected, clinical outcome was better in patients with bilateral PAE. However, approximately $50 \%$ of patients with unilateral embolization had a good clinical outcome, whereas $75 \%$ of patients with bilateral embolization reported a good clinical outcome. As previously shown [6, 7], there may exist anastomoses between prostatic arteries from both pelvic sides, which may partially explain these results. 
Overall, all of the evaluated parameters improved significantly after PAE. There was a mean IPSS improvement of 11.3 points $(44.8 \%$ ), and a mean QoL improvement of 1.93 points, with a prostate volume reduction of $19.2 \%$ and a PSA reduction of $30.9 \%$. Almost all of these changes were better after bilateral PAE; however, the differences between groups did not reach statistical significance. There was a greater IPSS/QoL improvement and PV reduction after bilateral PAE; however, the PSA reduction, the Qmax improvement, and the PVR reduction were slightly better after unilateral PAE (non significant differences). We believe that this is due to the fact that LUTS in $\mathrm{PE} / \mathrm{BPH}$ patients is very complex and may be caused by many factors. Also, there is a short follow-up time. However, the great variability of the results in both groups (unilateral and bilateral PAE) may be one of the main reasons why these differences did not reach statistical significance. Unilateral PAE may be one of many confounding factors that undermine the clinical outcome after $\mathrm{PAE}$ for PE/BPH.

The relief of the bladder obstruction did not follow the magnitude of clinical improvement (mean Qmax improvement of $4.0 \mathrm{~mL} / \mathrm{s}$; mean PVR reduction of $35.9 \mathrm{~mL}$ ). This is not surprising, because PAE does not remove prostatic tissue. The IIEF had a slight improvement (1.55 points) probably due to the discontinuation of all prostatic medication after PAE.

We were able to perform PAE as an outpatient procedure in almost $90 \%$ of patients with minimal pain associated and no vomiting or other postembolization symptoms. No major complications were reported in this study, and all minor complications could be addressed with medical/conservative care. Approximately $30-40 \%$ of patients experience a burning sensation in the urethra and irritative voiding (frequency, urgency) in the first 2 weeks after PAE. These symptoms may range from light to very severe and may raise the suspicion of a urinary tract infection (we only considered those cases with positive urine cultures). Patients should be warned that this is a normal finding after PAE and that is usually self-limited, disappearing after the first 2 weeks. We did not consider these symptoms as minor complications. We had approximately $10 \%$ of urinary tract infections despite the antibiotic coverage before and after PAE (with no differences between groups).

Up to $27.9 \%$ of patients had poor clinical outcome after PAE. If the Qmax criteria were neglected and only symptomatic parameters were evaluated (IPSS/QoL and the need of further prostatic treatment), poor outcome after PAE was observed in $21.3 \%$ patients. After unilateral PAE, $11 \%$ of patients $(n=1)$ had significant IPSS/QoL improvements and required no additional treatments but were considered to have poor outcome due to the Qmax criteria. The same applied to $28 \%$ of patients $(n=7)$ after bilateral PAE, with no significant differences between groups. Once more, the dissociation between clinical and urodynamic improvements partially explains these results. Most of the patients with poor clinical outcome remained under observation and resumed the prostatic medication with only six $(4.9 \%)$ patients needing to undergo surgery. The patients with good clinical outcome remained without any prostatic medication.

This study has limitations. The follow-up time is not very long (and different between groups), and we did not compare PAE with other therapeutical options or placebo. There were no pressure-flow urodynamics performed (which may help to exclude patients with LUTS due to other causes such as bladder dysfunction), and the study was not randomized. The endpoint follow-up period in each group and between groups was not the same for all patients.

PAE is a safe and effective technique that can induce $45 \%$ improvement in the IPSS score and a prostate volume reduction of $19 \%$, with good clinical outcome in up to 75 $\%$ of treated patients. Bilateral PAE seems to lead to better clinical results; however, up to $50 \%$ of patients after unilateral PAE may have a good clinical outcome.

Conflict of interest Tiago Bilhim and Hugo Rio Tinto-Cook Medical (speaker/honoraria; consultant/advisory board). João Pisco, Lúcia Fernandes, Luís Campos Pinheiro, Marisa Duarte, José A. Pereira, António G. Oliveira and João O’Neill—no conflict of interest.

\section{References}

1. DeMeritt JS, Elmasri FF, Esposito MP et al (2000) Relief of benign prostatic hyperplasia-related bladder outlet obstruction after transarterial polyvinyl alcohol prostate embolization. J Vasc Interv Radiol 11(6):767-770

2. Carnevale FC, Antunes AA, da Motta Leal Filho JM et al (2010) Prostatic artery embolization as a primary treatment for benign prostatic hyperplasia: preliminary results in two patients. Cardiovasc Intervent Radiol 33(2):355-361

3. Pisco JM, Pinheiro LC, Bilhim T et al (2011) Prostatic arterial embolization to treat benign prostatic hyperplasia. J Vasc Interv Radiol 22(1):11-19

4. Carnevale FC, da Motta-Leal-Filho JM, Antunes AA et al (2011) Midterm follow-up after prostate embolization in two patients with benign prostatic hyperplasia. Cardiovasc Intervent Radiol 34(6):1330-1333

5. Bilhim T, Casal D, Furtado A et al (2011) Branching patterns of the male internal iliac artery: imaging findings. Surg Radiol Anat 33(2):151-159

6. Bilhim T, Pisco JM, Furtado A et al (2011) Prostatic arterial supply: demonstration by multirow detector angio CT and catheter angiography. Eur Radiol 21(5):1119-1126

7. Bilhim T, Pisco JM, Tinto Rio et al (2012) Prostatic arterial supply: anatomic and imaging findings relevant for selective arterial embolization. J Vasc Interv Radiol 23(11):1403-1415 
8. Angle JF, Siddiqi NH, Wallace MJ et al (2010) Quality improvement guidelines for percutaneous transcatheter embolization: Society of Interventional Radiology Standards of Practice Committee. J Vasc Interv Radiol 21(10):1479-1486

9. Homma Y, Kawabe K, Tsukamoto T et al (1996) Estimate criteria for efficacy of treatment in benign prostatic hyperplasia. Int J Urol 3(4):267-273

10. Seki N, Kai N, Seguchi H et al (2006) Predictives regarding outcome after transurethral resection for prostatic adenoma associated with detrusor underactivity. Urology 67(2):306-310

11. Mitchell ME, Waltman AC, Athanasoulis CA et al (1976) Control of massive prostatic bleeding with angiographic techniques. J Urol 115(6):692-695

12. Pisco JM, Martins JM, Correia MG (1989) Internal iliac artery: embolization to control hemorrhage from pelvic neoplasms. Radiology 172(2):337-339

13. Nabi G, Sheikh N, Greene D et al (2003) Therapeutic transcatheter arterial embolization in the management of intractable haemorrhage from pelvic urological malignancies: preliminary experience and long-term follow-up. BJU Int 92(3):245-247

14. Rastinehad AR, Caplin DM, Ost MC et al (2008) Selective arterial prostatic embolization (SAPE) for refractory hematuria of prostatic origin. Urology 71(2):181-184
15. Delgal A, Cercueil JP, Koutlidis N et al (2010) Outcome of transcatheter arterial embolization for bladder and prostate hemorrhage. J Urol 183(5):1947-1953

16. Liguori G, Amodeo A, Mucelli FP et al (2010) Intractable haematuria: long-term results after selective embolization of the internal iliac arteries. BJU Int 106(4):500-503

17. Sun F, Sánchez FM, Crisóstomo V et al (2008) Benign prostatic hyperplasia: transcatheter arterial embolization as potential treatment-preliminary study in pigs. Radiology 246(3):783-789

18. Jeon GS, Won JH, Lee BM et al (2009) The effect of transarterial prostate embolization in hormone-induced benign prostatic hyperplasia in dogs: a pilot study. J Vasc Interv Radiol 20(3):384-390

19. Sun F, Sánchez FM, Crisóstomo V et al (2011) Transarterial prostatic embolization: initial experience in a canine model. Am J Roentgenol 197(2):495-501

20. Mauro MA (2008) Can hyperplastic prostate follow uterine fibroids and be managed with transcatheter arterial embolization? Radiology 246(3):657-658 